\title{
Double-J Stenting Versus Percutaneous Nephrostomy; Postoperative Complications in Management of Ureteral Obstruction
}

\author{
Khalid Saeed ${ }^{1}$, Syed Atif Hussain², Shayan Rahim Kanjoo ${ }^{3}$, Bashir Ahmad ${ }^{4}$, Mujahid Hussain ${ }^{5}$
}

\author{
Associate Professor, Department of Urology, Sheikh Zayed Medical College (SZMC), Rahim Yar Khan Pakistan \\ Overall supervision, Manuscript writing, Reviewed the manuscript before submission for publication \\ 2 Assistant Professor, Department of Urology, Sheikh Zayed Medical College (SZMC), Rahim Yar Khan Pakistan \\ Data collection, Manuscript writing \\ 3 Senior Registrar, Department of Urology, Sheikh Zayed Hospital, Rahim Yar Khan Pakistan \\ 3 Data collection, Manuscript writing \\ 4 Emergency Medical Officer, THQ, Jampur Pakistan \\ 4 Data analysis, Manuscript writing \\ Assistant Professor, FG Degree College Kharian Cantt, Pakistan \\ Data analysis, Manuscript writing
}

\section{ABSTRACT}

Background: Ureteral obstruction is a common surgical problem and managed by stent insertion or percutaneous nephrostomy. However, there is still confusion that which procedure is better in terms of the complications. Objective: To compare the efficacy of double J stenting and percutaneous nephrostomy for ureteral obstruction in terms of post-operative complications. Study Design: Randomized Clinical Trial. Settings: Urology Department, Shaikh Zayed Hospital, Rahim Yar Khan-Pakistan. Duration: One years from January through December 2016. Methodology: Male/female patients (aged: 19-63 years) presenting in the OPD of the hospital with ureteral obstruction were registered during study period. Either of the groups i.e. A (double J stenting, $n=55$ ) or $B$ (percutaneous nephrostomy, $n=55$ ) was allotted to the subjects, randomly. The procedures and subsequent complications were addressed as per hospital protocols. Results: Most of the patients i.e., 75 (68.2\% of 110) were male whereas age statistics were, as: $M=49.47, S D=8.2$ (range 19-62) years. Ureteral stone(s) was found as the most prevalent cause of ureteral obstruction in group A $(49.1 \%, \mathrm{n}=27)$ and B $(40.0 \%, \mathrm{n}=22)$. Hematuria was seen as a common complication: $33.3(n=5)$ and $42.9 \%(n=3)$ in group A and B, respectively. The rate of complications was significantly lower in group B than A [12.7\% $(n=7)$ vs. $27.3 \%(n=15)$, respectively] $(p=.04)$. Conclusion: Percutaneous nephrostomy is a better procedure (in terms of post-operative complications) than double J stenting in management of ureteral obstruction.

Keywords: Ureteral obstruction, Percutaneous nephrostomy, Stents, Postoperative complication.

How to Cite: Saeed K, Hussain SA, Kanjoo SR, Ahmad B, Hussain M. Double-J Stenting Versus Percutaneous Nephrostomy; Postoperative Complications in Management of Ureteral Obstruction. APMC 2020;14(4):336-9. DOI: 10.29054/ APMC/2020.1108

\section{INTRODUCTION}

Ureteral obstruction ${ }^{1}$ i.e. any obstruction in the ureter(s) poses considerable threats for renal functions by painful blockage of urinary drainage. Uremia, water-electrolyte imbalance and urinary tract infections are amongst the most common clinical findings. The patient with reduced alertness is under consistent fatal consequences and financial pressures. Unluckily, Pakistan positioned $53^{\text {rd }}$ in the world with kidney diseases-based (including ureteral obstruction) mortality rate of 23.62 per 100,000 population. ${ }^{2}$

Ureteral obstruction is either malignant or benign in origin. Mostly, malignant obstruction is the resultant of intrinsic urologic malignancy ${ }^{3}$ e.g., prostate cancer. Similarly, the etiology of benign ureteral obstruction is generally a consequence of luminal pathology e.g., ureteral stones. Any clinician opts an optimal definitive emergency procedure of decompression (percutaneous nephrostomy/double J stenting) for symptomatic relief from urine obstruction and reversal of renal physiology. Moreover, measures are also taken to minimize further urologic intervention, hospitalization and adverse impacts of quality of life (QoL).4,5 Percutaneous nephrostomy (PCN) is minimally invasive ultrasonography-guided procedure $^{6}$ while double-J ureteral stenting (DJS) becomes choice for obstruction on large-sized ureteral stone.

Like any other interventional procedure, there are chances of post-operative complications. Hematuria ${ }^{7}$ and septicemia are well-known complications of double-J stents. Similarly, ureteral perforation or stent migration can also be expected. Similarly, PCN blockage ${ }^{8}$ is a common issue. The complications are then addressed, accordingly.

In case of management of ureteral obstruction, there is no strong evidence to support the superiority of one methodology (with respect to post-operative complications) over other between stenting and 
nephrostomy. 9,10 Similarly, there is no paper on such issue at study setting of present research. To fill the gap, present work was conducted with the aim: "To compare the efficacy of double J stenting and percutaneous nephrostomy for ureteral obstruction in term of postoperative complications". The results will open gates for further investigations towards validity of the optimal procedure.

\section{METHODOLOGY}

Study Design: Randomized controlled trial.

Settings: Department of Urology, Sheikh Zayed Hospital, Rahim Yar Khan Pakistan.

Duration: One year i.e., January through December 2016. Sample Technique: Consecutive sampling technique.

Sample Size: The total sample size of 110 (i.e., 55 for each of the two groups) emerged when values of Group A (anticipated incidence of hematuria/bleeding against double-J ureteral stenting) $=37 \%, 11$ and Group B (anticipated ... percutaneous nephrostomy tube insertion) $=11 \%,{ }^{1} \alpha=0.05, \beta=0.1$, and power of test $=$ $90 \%$ were inserted in the online sample size calculator.

Inclusion Criteria: All the male/female patients (aged: 19-63 years) presenting in the outdoor patient department of the study setting with benign/ malignant ureteral obstruction and hydronephrosis were registered.

Exclusion Criteria: The patients with bleeding diathesis, sepsis, anesthetic drug allergy, or uremia were excluded. Data Collection Procedure: It was initiated getting permission from the Institutional Review Board.

Written participation consent was taken before group allotment. Either of the groups i.e. A or B was allotted randomly to each of the recruiters by a biostatistician.

Clinical manifestations were collected before KUB (kidney, ureter, bladder) sonography. Single dose of prophylactic antibiotics was administered before intervention, intravenously. In group A, the double-J ureteral stent (6F with side holes) was inserted retrograde using aseptic cystoscopy technique under mild sedation/local anesthesia (instilling $2 \%$ xylocaine gel in urethra). In group $B$, the ultrasound-guided percutaneous nephrostomy tube was inserted at the puncture site, subcutaneously. The complications e.g. bleeding, hematuria and septicemia of both the groups were documented in immediate post-operative and follow up sessions (15 th and $30^{\text {th }}$ day) using the KUB sonography. Patients with complications were immediately managed as per hospital's protocols.

The data was analyzed in SPSS version 23.0. Quantitative or qualitative data were processed for mean (SD) or percentage (n), respectively. The success rate (in terms of complications) was associated with interventional procedure using chi-square test. Statistical significance was accepted at $\mathrm{P}<0.05$.

\section{RESULTS}

All the 110 subjects of both the groups i.e., group A (double-J stenting) and B (percutaneous nephrostomy) adhered with the therapies (response rate $=100 \%$ ). Most of the patients i.e., 75 (68.2\%) were male by gender. The SPSS-mediated processing of the data on biological age (confirmed by CNIC) of the patients at the time of registration revealed statistics, as: $M=49.47, S D=8.2$ (range 19-62) years. Most of the patients (i.e., $\mathrm{n}=102$, 92.7\%) were aged: 41 - 63 years.

Data presented in Table 1 show the incidence rate of different causes of the ureteric obstruction against the two study groups i.e. A and B. Ureteral stone(s) was found as the most prevalent cause in group A $(49.1 \%, \mathrm{n}=27)$ and B $(40.0 \%, \mathrm{n}=22)$ followed by PUJ - a genetic disorder obstruction (18.2 vs. $21.8 \%$, respectively). Endometriosismediated ureteral obstruction was seen in four $(7.3 \%)$ subjects of the group A patients. Contrary to it, incidence rate of the benign prostatic hyperplasia -based obstruction was comparatively higher in group B than A (12.7 vs. $7.3 \%$, respectively). Schistosomiasis (parasitic infection) caused obstruction in two and three patients of group $\mathrm{A}$ and $\mathrm{B}$, respectively.

Table 1: Causes of ureteric obstruction

\begin{tabular}{|l|c|c|}
\hline \multirow{2}{*}{\multicolumn{1}{|c|}{ Cause }} & \multicolumn{2}{c|}{ Study group; \% (n) } \\
\cline { 2 - 3 } & A (n = 55) & B (n = 55) \\
\hline PUJ obstruction & $18.2(10)$ & $21.8(12)$ \\
\hline Ureteral stone(s) & $49.1(27)$ & $40.0(22)$ \\
\hline Ureteral blood clot(s) & $3.6(2)$ & $7.3(4)$ \\
\hline $\begin{array}{l}\text { Tumor or retroperitoneal } \\
\text { fibrosis in abdomen }\end{array}$ & $9.1(5)$ & $5.5(3)$ \\
\hline Endometriosis (in females) & $7.3(4)$ & $0.0(0)$ \\
\hline $\begin{array}{c}\text { Swollen ureteric wall due to: } \\
\text { i. } \quad \begin{array}{c}\text { Tuberculosis } \\
\text { ii. Schistosomiasis }\end{array}\end{array}$ & $1.8(1)$ & $7.3(4)$ \\
\hline BPH (in males) & $7.3(4)$ & $12.7(7)$ \\
\hline
\end{tabular}

PUJ (pelvic ureteric junction), BPH (benign prostatic hyperplasia), No case with biofilm and bacterial (other than Mycoplasma spp.) colonization on DJ stent

Hematuria - blood in urine was found as the most prevalent post-operative complication with $33.3(\mathrm{n}=5)$ and $42.9 \%(\mathrm{n}=3)$ rate in group $\mathrm{A}$ and $\mathrm{B}$, respectively as displayed in Table 2. It was followed by septicemia along with fever (26.7\% against A group while $28.6 \%$ in B). In group A, procedural failure emerged in one $(6.7 \%)$ of the subject patients. There were some group-specific outcomes as trigone irritation with pain, ureteral perforation or stent migration were seen in only group A while PCN dislodgement/blockage or injuries to adjacent organs confined to group B. 
Table 2: Post-operative complications in patients with ureteral obstruction

\begin{tabular}{|l|c|c|}
\hline \multirow{2}{*}{\multicolumn{1}{|c|}{ Complication }} & \multicolumn{2}{c|}{ Study group; \% (n) } \\
\cline { 2 - 3 } & A (n = 15) & B (n = 7) \\
\hline Septicemia and fever & $26.7(4)$ & $28.6(2)$ \\
\hline Hematuria & $33.3(5)$ & $42.9(3)$ \\
\hline Trigone irritation with pain & $13.3(2)$ & ---- \\
\hline PCN dislodgement/blockage & --- & $14.3(1)$ \\
\hline Ureteral perforation & $6.7(1)$ & --- \\
\hline Stent migration & $6.7(1)$ & --- \\
\hline Injuries to adjacent organs & --- & $14.3(1)$ \\
\hline Stent encrustation & $6.7(1)$ & --- \\
\hline Procedural failure & $6.7(1)$ & $0.0(0)$ \\
\hline
\end{tabular}

--- (Not applicable)

Post-operative complications appeared in 15 (i.e., 27.3\%) patients of group A (success rate $=72.7 \%$ ) in $1^{\text {st }}$ phase of the outcomes. It decreased to 7 (i.e., 12.7\%) patients with success rate of $87.3 \%$ in B as shown in bar graph (Fig. 1) after $2 \times 2$ crosstabulation. There was significant difference between the two success rates $(p=.04)$ when data was processed in chi-square test. Management of the complications (as per hospital protocols) led to reporting of complicated cases, as: Group A $=$ one $(6.7 \%$; success rate raised from 72.7 to $98.2 \%)$ while $\mathrm{B}=0(0.0 \%$; success rate shifted from 87.3 to $100 \%$ ) in $2^{\text {nd }}$ phase of outcomes.

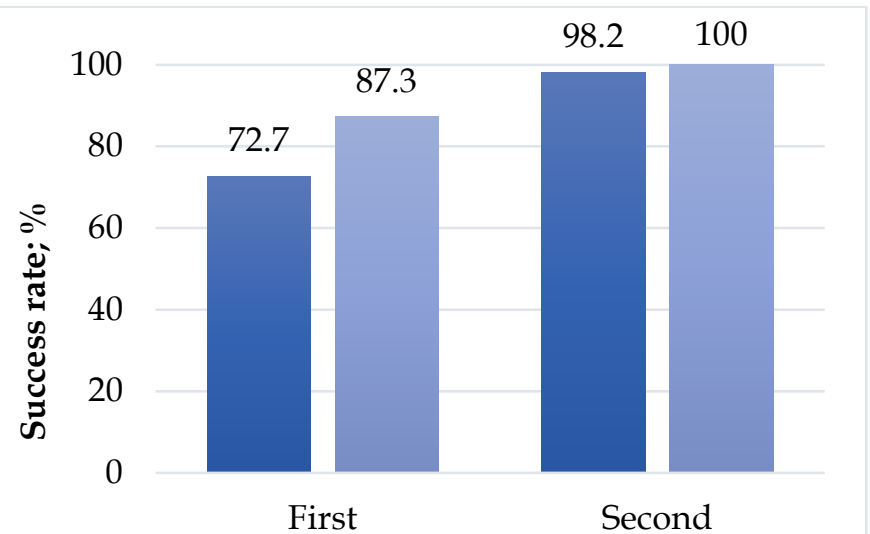

\section{Phase of Outcome - Group A Group B}

Figure 1: Success rate of the therapies for ureteral obstruction in two phases of outcome

$\mathrm{n}=55$ for each of the two study groups: A (DJ stenting) and B (PC nephrostomy)

\section{DISCUSSION}

Adherence with therapy in all the study subjects (response rate $=100 \%$ ) is a positive approach as it ensures speed cost-effective recovery and improves quality of life e.g., after indwelling stent placement ${ }^{12}$ on ureteral obstruction. The mean age of the participants $(49.47$ years) is slightly higher than a published study ${ }^{13}(45.0$ years). The age difference seems to be the consequent of chance sampling and/or moderating factors including genetics and life style.

Kidney stone is one of the major causes of ureteral obstruction. ${ }^{14}$ Our findings are in accordance with perception as their incidence rate is between 40 to $50 \%$. Whereas, $2^{\text {nd }}$ highest positioning of pelvic ureter junction disorder is alarming one as it has genetic background. On the other side, ureteral infections like tuberculosis or schistosomiasis (parasitic infection) can be seen in many cases of ureteral obstruction as in present study (1 to 4 cases against any group). The unmanaged tuberculosis can cause death. ${ }^{15}$ Opposite to a study, ${ }^{16}$ no case of biofilm and bacterial (other than Mycoplasma spp.) colonization on DJ stent is plus point of the procedure. Accountable rate $(3-5 \%)$ of tumor or retroperitoneal fibrosis in abdomen - a causative morbidity of ureteral obstruction is significant in management. However, precise expertise is expected in identification of the tumor as primary retroperitoneal paraganglioma can mimic a ureteral tumor. ${ }^{17}$

Despite of equal effective of the DJS and PCN for ureteral stones in normalizing urea and creatinine, ${ }^{18}$ there is big chance of appearance of post-operative complications in the patients who underwent interventional management of ureteral obstruction. Unfortunately, hematuria is common one them. ${ }^{19}$ Our results (33.3 vs. $42.9 \%$ against DCN and PCN groups, respectively) are in line with that perception. Infection-mediated septicemia and allied mild to severe fever ranked $2^{\text {nd }}$ to hematuria because nonintentional mishandling in procedure is its root cause. Finding of higher incidence rate of septicemia in PCN than DJS is in accordance with a previous similar work ${ }^{20}$ indicating some loopholes in the PCN. Stent migration ${ }^{21}$ or encrustation can be expected in DJS for ureteral obstruction but total failure of this procedure is a rare one while advanced stage of the causative agent e.g., malignancy cannot be ruled out in the context. The failure needs readdress of the issue for complete relief otherwise patient shifts to bad experience of hemodialysis ${ }^{22}$ using various devices. In case of small-sized stone, medical expulsive therapy can be tried, effectively. ${ }^{23}$

Comparatively higher success rate (inverse of complication rate) against nephrostomy (87.3\%) than stenting $(72.7 \%)$ have same trends as in an already reported Pakistani study ${ }^{24}$ for the same objectives. Stent migration/encrustation and ureteral perforation are DJSspecific beside painful trigone irritation. Moreover, very slight difference $(1.8 \%)$ in the success rate after management of the complications in $2^{\text {nd }}$ phase of present study is attributed to the managemental skills of the healthcare professionals ${ }^{25}$ of the study setting. 


\section{CONCLUSION}

Percutaneous nephrostomy (PCN) showed better efficacy than double J stenting (DJS) in terms of postoperative complications for definitive management of ureteral obstruction caused by different factors including extrinsic/intrinsic malignancy.

\section{LIMITATIONS}

The study confined to pilot scale level on account of small sample size and certain constrains e.g. man power, and finances.

\section{SUGGESTIONS / RECOMMENDATIONS}

Further studies should be conducted at large scale to revalid the results of present study before recommendation of a suitable procedure for clinicians.

\section{CONFLICT OF INTEREST / DISCLOSURE}

None.

\section{ACKNOWLEDGEMENTS}

Authors are thankful to all the paramedics who made the conduct possible.

\section{REFERENCES}

1. Tan S, Tao Z, Bian X, Zhao Y, Wang N, Chen X, et al. Ureteral stent placement and percutaneous nephrostomy in the management of hydronephrosis secondary to cervical cancer. Eur J Obstet Gynecol Reprod Biol. 2019;241:99-103.

2. WHR (World Health Rankings) - 2018. https://www. worldlifeexpectancy. com/ cause-of-death/kidney- disease / bycountry/. Retrieved on Nov 22, 2020

3. Falzarano SM, Feely MM. How can biomarkers assist the prognosis of urologic malignancies? Expert Rev Mol Diagn. 2020;20(2):131-3.

4. Liu KL, Lee BC, Ye JD, Chang YH, Chang CC, Huang KH, et al. Comparison of single and tandem ureteral stenting for malignant ureteral obstruction: a prospective study of 104 patients. Eur Radiol. 2019;29(2):628-35.

5. Lupu S, Brînză A, Socea B, Marcu D, Peride I, Stanescu AMA, et al. A brief review of the literature on the malignant ureteral obstruction. J Mind Med Sci. 2018;5(2):189-94.

6. Georgescu D, Jecu M, Geavlete PA, Geavlete B. Percutaneous Nephrostomy. In: Percutaneous surgery of the upper urinary tract. Academic Press. 2016;15(1):159-63.

7. Jain R, Raju K, Bali RS, Chander J, Neogi S. Prognostic implications of double $\mathrm{J}$ ureteral stenting in patients with renal stones undergoing extracorporeal shockwave lithotripsy. Intl J Res Med Sci. 2017;5(9):3831.

8. Turo R, Horsu S, Broome J, Das S, Gulur DM, Pettersson B, et al. Complications of percutaneous nephrostomy in a district general hospital. Turk J Urol. 2018;44(6):478-83.

9. Betschart P, Zumstein V, Buhmann MT, Altenried S, Babst C, Müllhaupt G, et al. Symptoms associated with long-term double-J ureteral stenting and influence of biofilms. Urology. 2019;134:72-8.
10. Jairath A, Ganpule A, Desai M. Percutaneous nephrostomy step by step. Mini-invasive Surg. 2017;1:180-5.

11. Al-Hammouri F, Abo-Qamar A, Al-Majali A. Obstructive uropathy treatment complication rate after percutaneous nephrostomy versus double J catheter insertion. J Royal Med Serv. 2018;25(2):4653.

12. Li Z, Sheng Y, Wang S, Liu Z, Xia B, Wang J. Effect of potassium sodium hydrogen citrate granules on preventing adherent stones on ureteral stent after retrograde intrarenal surgery. World J Urol. 2020;5:1-2.

13. Aghamir SMK, Heidari R, Bayesh S, Salavati A, Elmimehr R. Are Nephrostomy and ureteral stent necessary after multi-access percutaneous nephrolithotomy? Current Urol. 2019; 13(3):141-4.

14. Uçar M, Özen O. The Relationship between ureteral obstruction time and secondary signs in computed tomography due to ureteral stone obstruction. J Coll Physicians Surg Pak. 2020;30(7):678-82.

15. Beaumier M, Roger H, Sauneuf B, Dugardin F, Camparo P, Queffeulou G. Disseminated tuberculosis revealed by obstructive and prerenal acute kidney failure. Nephrol Ther. 2019;15(3):169-73.

16. Shaikh GS, Shar AH, Imran M, Shaikh AA, Shaikh NA, Jalbani MH. Detection of biofilm and bacterial colonization on DJ stent in obstructive uropathy. Rawal Med J. 2020;45(2):350-2.

17. Sahu P, Singh S, Ahuja A, Sharma U, Mattoo S, Bhardwaj M. Unusual and rare cause of PUJ obstruction with a brief review of the literature. J Clin Urol. 2020;23(7):205141582095899.

18. Ali L, Khan S, Khan FH, Orakzai N. Comparison of percutaneous nephrostomy and double $\mathrm{j}$ stent in early normalization of renal function tests in patients with obstructive uropathy due to urolithiasis. Ann Pak Inst Med Sci. 2020;16(1):10-14.

19. Shoshany O, Erlich T, Golan S, Kleinmann N, Baniel J, Rosenzweig B, Eisner A, et al. Ureteric stent versus percutaneous nephrostomy for acute ureteral obstruction - clinical outcome and quality of life: a bi-center prospective study. BMC Urol. 2019;19(1):79.

20. Weltings S, Schout BMA, Roshani H, Kamphuis GM, Pelger RCM. Lessons from Literature: Nephrostomy Versus Double J Ureteral Catheterization in Patients with Obstructive Urolithiasis - Which Method Is Superior? J Endourol. 2019;33(10):777-86.

21. Zhu H, Wang J, Deng Y, Haung L, Zhu X, Dong J, et al. Use of double-J ureteric stents post-laparoscopic pyeloplasty to treat ureteropelvic junction obstruction in hydronephrosis for pediatric patients: a single-center experience. J Int Med Res. 2020;48(4):300060520918781.

22. Shafique MN, Akhtar SH, Mahnoor M, Hussain M. Hemodialysis Internal jugular vein versus Subclavian vein Catheters: Complications, patients' comfort, tolerance and cost-effectiveness. Pak J Med Sci. 2019;35(1):124-8.

23. Shafique MN, Hussain M. Efficacy of tamsulosin alone versus tamsulosin phloroglucinol combination therapy for medical expulsion of lower uretheral calculi. Paj J Med Sci. 2018;34(2):3938.

24. Ahmad I, Pansota MS, Tariq M, Shahzad MS, Ali ST, Hussain A. Comparison between double J (DJ) ureteral stenting and percutaneous nephrostomy $(\mathrm{PCN})$ in obstructive uropathy. Pak J Med Sci. 2013;29(3):725-9.

25. Panach-Navarrete J, Tonazzi-Zorrilla R, Martínez-Jabaloyas JM. Dislodgement in long-term patients with nephrostomy tube: Risk factors and comparative analysis between two catheter designs. J Endourol. 2020;3(2):227-32. 